\title{
A clinical prediction rule for perioperative mortality and major morbidity after laparoscopic giant paraesophageal hernia repair
}

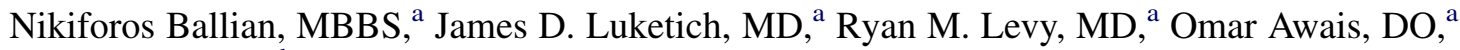 \\ Dan Winger, MS, ${ }^{\mathrm{b}}$ Benny Weksler, MD, ${ }^{\mathrm{a}}$ Rodney J. Landreneau, MD, ${ }^{\mathrm{a}}$ and Katie S. Nason, MD, MPH
}

\begin{abstract}
Objective: In the current era, giant paraesophageal hernia repair by experienced minimally invasive surgeons has excellent perioperative outcomes when performed electively. However, nonelective repair is associated with significantly greater morbidity and mortality, even when performed laparoscopically. We hypothesized that clinical prediction tools using pretreatment variables could be developed that would predict patientspecific risk of postoperative morbidity and mortality.
\end{abstract}

Methods: We assessed 980 patients who underwent giant paraesophageal hernia repair (1997-2010; 80\% elective and $97 \%$ laparoscopic). We assessed the association between clinical predictor covariates, including demographics, comorbidity, and urgency of operation, and risk for in-hospital or 30-day mortality and major morbidity. By using forward stepwise logistic regression, clinical prediction models for mortality and major morbidity were developed.

Results: Urgency of operation was a significant predictor of mortality (elective $1.1 \%$ [9/778] vs nonelective 8\% [16/199]; $P<.001$ ) and major morbidity (elective $18 \%$ [143/781] vs nonelective $41 \%$ [81/199]; $P<.001$ ). The most common adverse outcomes were pulmonary complications $(n=199 ; 20 \%)$. A 4-covariate prediction model consisting of age 80 years or more, urgency of operation, and 2 Charlson comorbidity index variables (congestive heart failure and pulmonary disease) provided discriminatory accuracy for postoperative mortality of $88 \%$. A 5-covariate model (sex, age by decade, urgency of operation, congestive heart failure, and pulmonary disease) for major postoperative morbidity was $68 \%$ predictive.

Conclusions: Predictive models using pretreatment patient characteristics can accurately predict mortality and major morbidity after giant paraesophageal hernia repair. After prospective validation, these models could provide patient-specific risk prediction, tailored for individual patient characteristics, and contribute to decisionmaking regarding surgical intervention. (J Thorac Cardiovasc Surg 2013;145:721-9)

Giant paraesophageal hernias (GPEHs) can be asymptomatic, cause chronic low-grade symptoms, or present acutely. Chronic GPEH-related symptoms, such as heartburn, chest discomfort, and dyspnea, are clear indications for surgical repair, which can be approached laparoscopically with acceptably low rates of mortality and morbidity when performed electively. ${ }^{1-4}$ Quality of life after laparoscopic repair is excellent, with relief of heartburn, dysphagia,

\footnotetext{
From the Division of Thoracic and Foregut Surgery, ${ }^{\mathrm{a}}$ Department of Cardiothoracic Surgery, University of Pittsburgh, Pittsburg, Pa; and University of Pittsburgh Clinical and Translational Science Institute, ${ }^{\mathrm{b}}$ Pittsburgh, Pa.

Grant Support: The project described was supported by Award Numbers K07CA151613 (KSN), UL1 RR024153, and UL1TR000005 from the National Cancer Institute. The content is solely the responsibility of the authors and does not necessarily represent the official views of the National Cancer Institute or the National Institutes of Health.

Disclosures: Authors have nothing to disclose with regard to commercial support.

Read at the 38th Annual Meeting of The Western Thoracic Surgical Association, Maui, Hawaii, June 27-30, 2012.

Received for publication June 10, 2012; revisions received Oct 10, 2012; accepted for publication Dec 10, 2012; available ahead of print Jan 14, 2013.

Address for reprints: Katie S. Nason, MD, MPH, 5200 Center Ave, Suite 715, Shadyside Medical Building, Pittsburgh, PA 15232 (E-mail: nasonks@upmc.edu). $0022-5223 / \$ 36.00$

Copyright $(2013$ by The American Association for Thoracic Surgery http://dx.doi.org/10.1016/j.jtcvs.2012.12.026
}

regurgitation, and dyspnea symptoms in the majority of patients. Preoperative pulmonary function abnormalities and anemia are also improved after GPEH repair. ${ }^{5-9}$ The improved perioperative mortality and morbidity associated with elective laparoscopic GPEH repair has prompted ongoing debate regarding the appropriate timing for repair and the safety of watchful waiting. ${ }^{10-12}$ Proponents of watchful waiting cite evidence that the historical risks, including high mortality and morbidity associated with gastric volvulus and strangulation, are overestimated. ${ }^{13}$ However, multiple retrospective reports have shown that mortality and major morbidity associated with nonelective GPEH repair was significantly higher than after elective GPEH repair. ${ }^{14,15}$ Clearly, the ability to weigh potential risks and benefits of operative intervention would be extremely useful in guiding treatment decisions.

Clinical prediction rules allow clinicians to determine the probability of an outcome using existing clinical covariates, either for their own decision-making or in counseling patients. ${ }^{16}$ For example, the Pneumonia Severity Index, developed to determine allocation of care in the treatment of community-acquired pneumonia, uses clinical variables obtained at emergency department presentation to assign 


\section{Abbreviations and Acronyms}

$\mathrm{CCI}=$ Charlson comorbidity index

$\mathrm{GPEH}=$ giant paraesophageal hernia

$\mathrm{IQR}=$ interquartile range

an index risk score. ${ }^{17}$ The patient's score guides clinical decisions regarding hospital admission and oral versus intravenous antibiotics. Similar clinical prediction rules to assign risk categories for mortality and major morbidity after GPEH repair could inform provider decisions regarding surgical intervention.

We hypothesized that clinical risk-stratification tools based on known preoperative covariates could provide patient-specific perioperative mortality and major morbidity estimates in the preoperative setting. The aim of this study was to develop easy-to-use clinical prediction rules, incorporating readily available preoperative covariates that have discriminatory accuracy for predicting patient risk for perioperative mortality and major morbidity after laparoscopic repair of GPEH.

\section{MATERIALS AND METHODS \\ Patient Selection}

Adult patients $(\mathrm{n}=980)$ who underwent primary transabdominal (laparoscopic or open) repair of GPEH (January 1, 1997, to August 31, 2010) were retrospectively identified from a prospectively maintained database. GPEH was defined as more than $30 \%$ gastric herniation into the posterior mediastinum. ${ }^{18}$ Patients undergoing elective $(\mathrm{n}=781 ; 80 \%)$ and nonelective surgery were included. Nonelective surgery was defined as urgent $(\mathrm{n}=173$; patient requiring admission for GPEH symptom management and repaired during the same admission) or emergency $(\mathrm{n}=26$; immediate operation in patient with acute GPEH-related complications). Patients with prior antireflux surgery or GPEH repair were excluded. This study was approved by our institutional review board (Pittsburgh, Pa).

\section{Operative Approach}

A laparoscopic approach was planned in 951 patients (97\%). Our approach to laparoscopic GPEH repair has been described. ${ }^{1,19}$ Hernia reduction, extensive esophageal mobilization, and crural reapproximation were performed in all patients. Definitive GPEH repair, with an antireflux procedure to minimize postoperative reflux, was performed in 888 patients $(91 \%)$. Fundoplication was partial (Toupet or Dor) in 206 patients and circumferential (Nissen) in the remaining 682 patients. Of the 888 patients treated with fundoplication, a Collis gastroplasty for esophageal lengthening was added in 465 patients $(52 \%)$. Mesh repair was performed in 116 patients $(12 \%)$. Patients who received GPEH repair without fundoplication were also included $(n=92[9 \%]$; eg, gastropexy, Roux-en-Y near-esophagojejunostomy, and gastroesophageal resection). Type of fundoplication and the need for mesh cruroplasty or esophageal lengthening were determined intraoperatively. GPEH repairs were performed by 19 surgeons over the time period of study with 498 $(51 \%)$ performed by the senior surgeon (JDL).

\section{Data Collection and Outcome Measures}

Identification of predictor covariates. Predictor variables were defined as patient demographics, symptoms, covariates defined by the Charlson comorbidity index $(\mathrm{CCI}),{ }^{20}$ urgency of operation, and laboratory and radiographic findings that were known when the decision for surgery was made. Covariates in the CCI that were present in less than $3 \%$ of patients were grouped together as 1 variable (rare CCI conditions). These included diabetes mellitus with organ damage, peripheral vascular disease, renal disease, liver disease, hemiplegia or paraplegia, lymphoma, leukemia, metastatic cancer, and acquired immunodeficiency syndrome.

Definition of outcome variables. Postoperative adverse outcomes, including hospital or 30-day mortality and major morbidity, were assessed using the Society of Thoracic Surgeons' definitions for postoperative complications. ${ }^{21} \mathrm{~A}$ binary outcome for mortality was defined as death during the initial hospital stay or within 30 days after surgery, whichever was longer. Likewise, a binary outcome measure for major morbidity was defined as at least 1 major adverse event during the initial hospital stay or within 30 days after surgery, whichever was longer, and included pneumonia, reintubation, tracheostomy, pulmonary embolism, myocardial infarction, congestive heart failure, acute renal failure, cerebral vascular accident, septic shock or bacteremia, postoperative gastric or esophageal leak, perioperative hernia recurrence, and readmission or reoperation within 30 days. Mortality data were missing for 3 patients who survived to discharge in less than 30 days but were subsequently lost to follow-up. Therefore, a total of 977 patients were assessed for predictors of mortality, and 980 patients were assessed for predictors of major morbidity.

\section{Statistical Analysis and Development of Clinical Prediction Rules}

Descriptive statistics were summarized with frequencies and percentages for categoric variables, and median with interquartile range (IQR) for continuous variables. The Fisher exact test and Student $t$ test were used to compare differences between groups. Predictive models for mortality and major morbidity were then derived using forward, stepwise logistic regression. The performance of each predictive model was then estimated using bootstrap leave-one-out cross-validation analysis. Statistical analysis was performed using IBM SPSS 19 (IBM Corp, Armonk, NY) and STATA SE 11.0 (StataCorp LP, College Station, Tex) software.

Because the number of patients with postoperative mortality was small $(\mathrm{n}=25)$, and thus limited the number of potential predictors that could be assessed, an a priori " $P$ value for inclusion" of .05 was chosen to indicate a statistically significant change in the model at each step in the regression. In addition, because urgency of operation is well established as a clinically important predictor of adverse outcome after GPEH repair, we began regression modeling for mortality by forcing the urgency variable into the model as the first covariate and then began forward, stepwise regression from that point. At each step, 1 additional variable was assessed using logistic regression; if the addition of that variable produced a significant change $(P=.05$ or less) in the predictive accuracy of the model, that variable was included and the next step was performed by re-running the logistic regression with the next variable. This process continued until no more variables could be added, and at that point, the model was considered final. Variable inclusion order depended on the variable that caused the most significant model change, that is, the smallest $P$ value for that step.

An identical process was performed for the major morbidity prediction model; however, because the number of patients experiencing major morbidity was large $(\mathrm{n}=224)$, an a priori $P$ value of .15 was selected as the " $P$ value for inclusion" cutoff. This number of events allowed us to assess up to 22 variables in the model, enabling a larger $P$ value for inclusion and reducing the chance that important predictors would be overlooked.

Of 980 patients, $26(2.7 \%)$ were excluded from the regression analyses for mortality and $23(2.3 \%)$ were excluded from the regression analyses for morbidity because of 1 or more missing predictor variables in any of the potential predictors considered in the model. We also excluded peptic ulcer disease, a CCI variable, from the mortality model because of inconsistency in the documentation of peptic ulcer disease in the medical record. 
To determine the discriminatory accuracy of the predictive model, the area under the receiver operating characteristic curve was determined for each outcome model. Risk scores for mortality and major morbidity were then calculated. By using the same method developed to assign risk score in the $\mathrm{CCI},{ }^{20}$ a risk score for each covariate in the model was determined by assessing the odds ratio and assigning a whole number points value. As was done in the original description of the CCI, for odds ratios 1.2 or greater and less than $1.5,1$ point was assigned; for odds ratios 1.5 or greater and less than $2.5,2$ points were assigned; for odds ratios 2.5 or greater and less than $3.5,3$ points were assigned and so on. Categories were then created on the basis of the risk score with 3 groups for the mortality risk model and 4 groups for the major morbidity risk model. The rate of mortality and major morbidity for each group was determined.

\section{RESULTS}

The majority of patients were female $(\mathrm{n}=733 ; 75 \%)$ with a median age of 71 years (IQR, 62-78 years). The median body mass index was $29 \mathrm{~kg} / \mathrm{m}^{2}$ (IQR, 26-33). At least $50 \%$ of the stomach was herniated into the mediastinum in $82 \%$ of patients and $24 \%$ had a completely intrathoracic stomach (Table 1). At least 1 preoperative CCI comorbidity was present in $60 \%$ of patients $(n=592)$. The most common comorbid condition was pulmonary disease. The most common preoperative symptoms were respiratory problems (dyspnea, recurrent pneumonia, or aspiration), chest or abdominal pain, heartburn, and regurgitation (Table 1).

\section{Predictors of Mortality and Major Morbidity}

In-hospital or 30-day mortality occurred in $2.6 \%$ of patients $(\mathrm{n}=25)$. Mortality was $1.1 \%$ after elective surgery and $8.0 \%$ after nonelective surgery $(P<.01)$. Additional factors associated with mortality included older age at operation, lower body mass index, history of congestive heart failure, cerebral vascular accident, dementia, pulmonary disease, peptic ulcer disease, and malignancy within the past 5 years. There was a trend toward increased mortality in patients with regurgitation as a presenting symptom (Table 1).

At least 1 major morbidity was identified in $22.9 \%$ of patients $(n=224)$. Older age at operation, lower body mass index, and larger preoperative hernia were significantly associated with an increased rate of major postoperative morbidity (Table 1). CCI variables associated with major postoperative morbidity include history of myocardial infarction or coronary revascularization, congestive heart failure, cerebral vascular accident or transient ischemic attack, dementia, and pulmonary disease (Table 1). The only preoperative symptom that was significantly associated with adverse outcome was heartburn, which was less likely to be present in patients who had at least 1 adverse event (Table 1). Patients with preoperative heartburn were also younger $(P<.001)$, more likely to have smaller hernia $(P<.001)$, and less likely to have urgent operation $(P<.001)$.

\section{Development of the Clinical Prediction Rule}

Forward stepwise logistic regression was performed to identify combinations of variables most predictive of postoperative mortality and major morbidity. For postoperative mortality, a 4 -variable model consisting of 2 CCI variables (history of congestive heart failure and history of pulmonary disease), age at operation ( $\geq 80$ vs $<80$ years), and urgency of operation (Table 2; risk score range, 0-20) predicted mortality with discriminatory accuracy of $88.4 \%$ (95\% confidence interval, 82.0-94.8). Patients were then categorized into groups on the basis of the mortality risk score. Low-risk (mortality risk score, 0-2), intermediate-risk (mortality risk score 3), and high-risk (mortality risk score, $4+$ ) categories were associated with increasing risk for postoperative mortality (Figure 1).

For postoperative morbidity, a 5-variable model consisting of sex, age at operation (by decade), history of congestive heart failure, history of pulmonary disease, and urgency of operation (Table 2; risk score range, 0 -12) predicted major morbidity with discriminatory accuracy of $67.8 \%$ (area under the receiver operating characteristic curve, 0.678; $95 \%$ confidence interval, 0.637-0.718). When risk score was categorized into minimal, low, intermediate, and high risk, increasing risk category was associated with increasing incidence of at least 1 major adverse outcome. For patients in the minimal risk category (morbidity risk score $0-2)$, at least 1 major adverse outcome was identified in $13.5 \%$ compared with a rate of $40.6 \%$ in the patients in the high-risk category (morbidity risk score $5+$ ). Increasing risk category was also associated with progressively increasing rates of most of the major adverse outcomes assessed in the study (Table 3).

To assess how the models developed for major adverse outcome and postoperative mortality might generalize to an independent data set, we performed leave-one-out bootstrap cross-validation. The leave-one-out cross validation error rate was $21.503 \%$ for any major adverse outcome and $2.304 \%$ for postoperative mortality. These are nearly identical to the $21.399 \%$ and $2.304 \%$ error rates from the fitted models, indicating that application of the models to new patient data sets would yield similar results.

\section{Factors Associated With Nonelective Operation}

Because nonelective operation was a significant, independent predictor of mortality and major morbidity in our models, we assessed the clinical covariates associated with urgent or emergency GPEH repair (Table 4). Patients undergoing nonelective operation were more likely to be male, to be in the 70+-year age groups, to be underweight or ideal body weight, and to have larger hernia. Charlson comorbid diseases were more common in patients who underwent nonelective repair. Symptoms that were more commonly present in patients who underwent nonelective repair included respiratory symptoms, chest or abdominal pain, heartburn, regurgitation, and bloating. Age 80 years or more at operation, preoperative hernia size of $75 \%$ or greater, and a history of dementia or peptic ulcer disease 
TABLE 1. Association of patient demographics, comorbid diseases, and symptoms with major adverse outcome and mortality after giant paraesophageal hernia repair

\begin{tabular}{|c|c|c|c|c|c|c|c|c|}
\hline \multirow{3}{*}{ Patient demographics: $n(\%)$} & & \multirow[b]{2}{*}{ Overall } & \multicolumn{2}{|c|}{ Major adverse outcome } & \multirow{3}{*}{$P$ value } & \multicolumn{3}{|c|}{ 30-d mortality } \\
\hline & & & \multirow{2}{*}{$\begin{array}{c}\text { No } \\
\mathrm{n}=\mathbf{7 5 6}\end{array}$} & \multirow{2}{*}{$\begin{array}{c}\text { Yes } \\
\mathrm{n}=\mathbf{2 2 4}\end{array}$} & & \multirow{2}{*}{$\begin{array}{c}\text { Alive } \\
\mathrm{n}=952\end{array}$} & \multirow{2}{*}{$\begin{array}{c}\text { Dead } \\
n=25\end{array}$} & \multirow[b]{2}{*}{$P$ value } \\
\hline & & $\mathbf{n}=\mathbf{9 8 0}$ & & & & & & \\
\hline \multirow[t]{2}{*}{ Sex } & Female & $733(75)$ & $574(78)$ & $159(22)$ & .137 & $713(78)$ & $18(2)$ & .815 \\
\hline & Male & $247(25)$ & $182(74)$ & $65(26)$ & & $239(97)$ & $7(3)$ & \\
\hline \multirow[t]{5}{*}{ Age group at operation } & $<50 \mathrm{y}$ & $60(6)$ & $53(88)$ & $7(12)$ & $<.001$ & $59(98.3)$ & $1(1.7)$ & $<.001$ \\
\hline & $50-59$ y & $148(15)$ & $124(84)$ & $24(16)$ & & $147(100)$ & $0(0)$ & \\
\hline & $60-69$ y & $261(27)$ & $212(81)$ & $49(19)$ & & $260(99.6)$ & $1(0.4)$ & \\
\hline & $70-79$ y & $319(32)$ & $245(77)$ & $74(23)$ & & 315 (98.7) & $4(1.3)$ & \\
\hline & $\geq 80 \mathrm{y}$ & $192(20)$ & $122(64)$ & $70(36)$ & & $171(90)$ & $19(10)$ & \\
\hline \multirow{5}{*}{ BMI by WHO classification* } & Underweight & $16(2)$ & $10(63)$ & $6(37)$ & .042 & $14(87.5)$ & $2(12.5)$ & .001 \\
\hline & Ideal & $185(19)$ & $131(71)$ & $54(29)$ & & $175(94.6)$ & $10(5.4)$ & \\
\hline & Overweight & $356(37)$ & $281(79)$ & $75(21)$ & & $345(97.7)$ & $8(2.3)$ & \\
\hline & Obese & $258(27)$ & $210(81)$ & $48(19)$ & & $257(99.6)$ & $1(0.4)$ & \\
\hline & Severely obese & $149(15)$ & $119(80)$ & $30(20)$ & & $148(99.3)$ & $1(0.7)$ & \\
\hline \multirow[t]{4}{*}{ Preoperative hernia size $(\%) \dagger$} & $30 \%-49 \%$ & $174(18)$ & $146(84)$ & $28(16)$ & .009 & $169(97.1)$ & $5(2.9)$ & .171 \\
\hline & $50 \%-74 \%$ & $354(36)$ & $276(78)$ & $78(22)$ & & $345(97.7)$ & $8(2.3)$ & \\
\hline & $75 \%-99 \%$ & $212(22)$ & $166(78)$ & $46(22)$ & & $210(99.1)$ & $2(0.9)$ & \\
\hline & Intrathoracic Stomach & $240(24)$ & $168(70)$ & $72(30)$ & & $228(95.8)$ & $10(4.2)$ & \\
\hline \multicolumn{9}{|c|}{ History of pretreatment Charlson-defined patient comorbidity } \\
\hline \multirow[t]{2}{*}{ Myocardial infarction or revascularization } & No & $833(85)$ & $653(78)$ & $180(22)$ & .033 & $812(97.6)$ & $20(2.4)$ & .402 \\
\hline & Yes & $147(15)$ & $103(70)$ & $44(30)$ & & $140(96.5)$ & $5(3.5)$ & \\
\hline \multirow[t]{2}{*}{ Congestive heart failure } & No & $941(96)$ & $741(79)$ & $200(21)$ & $<.001$ & $918(97.9)$ & $20(2.1)$ & .002 \\
\hline & Yes & $39(4)$ & $15(38)$ & $24(62)$ & & $34(87.2)$ & $5(12.8)$ & \\
\hline \multirow[t]{2}{*}{ Cerebral vascular accident or transient attack } & No & $904(92)$ & $708(78)$ & $196(22)$ & .004 & 882 (97.9) & $19(2.1)$ & .010 \\
\hline & Yes & $76(8)$ & $48(63)$ & $28(37)$ & & $70(92.2)$ & $6(7.9)$ & \\
\hline Dementia & No & $937(96)$ & $732(78)$ & $205(22)$ & .001 & 917 (98.2) & $17(1.8)$ & $<.001$ \\
\hline & Yes & $43(4)$ & $24(56)$ & $19(44)$ & & $35(81.4)$ & $8(18.6)$ & \\
\hline Pulmonary disease & No & $690(70)$ & $548(79)$ & $142(21)$ & .010 & $675(98.3)$ & $12(1.8)$ & .024 \\
\hline & Yes & $290(30)$ & $208(72)$ & $82(28)$ & & $277(95.5)$ & $13(4.5)$ & \\
\hline Connective tissue disorder & No & $942(96)$ & $728(77)$ & $214(23)$ & .560 & $916(97.6)$ & $23(2.5)$ & .253 \\
\hline & Yes & $38(4)$ & $28(74)$ & $10(26)$ & & $36(94.7)$ & $2(2.3)$ & \\
\hline Peptic ulcer disease & No & $794(81)$ & $617(78)$ & $177(22)$ & .384 & 777 (98.2) & $14(1.8)$ & .003 \\
\hline & Yes & $186(19)$ & $139(18)$ & $47(21)$ & & $175(94.1)$ & $11(5.9)$ & \\
\hline Diabetes requiring medical therapy & No & 909 (93) & 705 (78) & $204(22)$ & .304 & $884(97.6)$ & $22(2.4)$ & .419 \\
\hline & Yes & $71(7)$ & $51(72)$ & $20(28)$ & & $68(95.8)$ & $3(4.2)$ & \\
\hline Malignancy in the past $5 \mathrm{y}$ & No & $924(94)$ & $716(77)$ & $208(23)$ & .325 & $900(97.7)$ & $21(2.3)$ & .050 \\
\hline & Yes & $56(6)$ & $40(71)$ & $16(29)$ & & $52(92.9)$ & $4(7.1)$ & \\
\hline Rare CCI variables $\ddagger$ & No & $939(96)$ & $730(78)$ & $209(22)$ & .038 & $914(97.7)$ & $22(2.4)$ & .083 \\
\hline & Yes & $41(4)$ & $26(63)$ & $15(37)$ & & 38 (92.7) & $3(7.3)$ & \\
\hline Preoperative symptoms & & & & & & & & \\
\hline Respiratory symptoms & No & $232(34)$ & $183(79)$ & $49(21)$ & .443 & $223(96.1)$ & $9(3.9)$ & .341 \\
\hline & Yes & $450(66)$ & $342(76)$ & $108(24)$ & & $436(97.5)$ & $11(2.5)$ & \\
\hline Chest or abdominal pain & No & $273(32)$ & $221(81)$ & $52(19)$ & .081 & $266(97.8)$ & $6(2.2)$ & 1.000 \\
\hline & Yes & $589(68)$ & $444(75)$ & $145(25)$ & & $574(97.8)$ & $13(2.2)$ & \\
\hline Heartburn & No & $335(35)$ & $233(70)$ & $102(30)$ & $<.001$ & $323(97)$ & $10(3)$ & .365 \\
\hline & Yes & $626(65)$ & $513(82)$ & $113(18)$ & & $613(98.1)$ & $12(1.9)$ & \\
\hline Dysphagia & No & $533(55)$ & $406(76)$ & $127(24)$ & .353 & $519(97.6)$ & $13(2.4)$ & 1.000 \\
\hline & Yes & $431(45)$ & $340(79)$ & $91(21)$ & & $418(97.4)$ & $11(2.6)$ & \\
\hline Regurgitation & No & 377 (39) & $286(76)$ & $91(24)$ & .345 & $370(98.7)$ & $5(1.3)$ & .061 \\
\hline & Yes & $590(61)$ & $463(78)$ & 127 (22) & & 569 (96.6) & $20(3.4)$ & \\
\hline Bloating & No & $619(66)$ & 476 (77) & $143(23)$ & .411 & 602 (97.6) & $15(2.4)$ & .649 \\
\hline & Yes & $325(34)$ & $258(79)$ & $67(21)$ & & $318(98.2)$ & $6(1.9)$ & \\
\hline
\end{tabular}

BMI, Body mass index $\left(\mathrm{kg} / \mathrm{m}^{2}\right) ; W H O$, World Health Organization; CCI, Charlson Comorbidity Index. *Underweight BMI <18.5, ideal BMI 18.5-25, overweight BMI 25-30, obese BMI 30-35, severely obese BMI $\geq 35$. BMI was available for 964 patients with 30 -day mortality data for 961 . $\dagger$ Percent of stomach within the mediastinum by barium esophagram, preoperative endoscopy, or intraoperative description. $\ddagger$ Rare CCI variables $(<3 \%$ of cohort) include peripheral vascular disease, renal disease, liver disease, diabetes mellitus with organ damage, hemiplegia or paraplegia, lymphoma, leukemia, metastatic cancer, and acquired immunodeficiency syndrome. 
TABLE 2. Clinical prediction rules for in-hospital or 30-day mortality (A, mortality model) and major morbidity (B, morbidity model): Variables included in predictive models after forward stepwise logistic regression analysis and points assigned for each risk factor present

\begin{tabular}{|c|c|c|c|c|c|}
\hline Variables in each model & Points & Comparison & $P$ value & Adjusted OR $\ddagger$ & $95 \% \mathrm{CI}$ \\
\hline \multicolumn{6}{|l|}{ A. Mortality } \\
\hline Congestive heart failure* & 5 & Present vs absent & .009 & 4.740 & $1.481-15.172$ \\
\hline Pulmonary disease* & 3 & Present vs absent & .009 & 3.342 & $1.345-8.306$ \\
\hline Surgery type $\dagger$ & 3 & Nonelective vs elective & .021 & 3.165 & $1.193-8.397$ \\
\hline Age $\geq 80(y)$ & 9 & $\geq 80$ vs $<80$ & $<.001$ & 8.577 & $3.043-24.174$ \\
\hline \multicolumn{6}{|l|}{ B. Morbidity } \\
\hline Sex & 1 & Male vs female & .122 & 1.328 & $0.927-1.901$ \\
\hline Congestive heart failure* & 4 & Present vs absent & $<.001$ & 4.267 & $2.083-8.737$ \\
\hline Pulmonary disease* & 2 & Present vs absent & .015 & 1.515 & $1.083-2.121$ \\
\hline Surgery type $\dagger$ & 2 & Nonelective vs elective & $<.001$ & 2.142 & $1.466-3.128$ \\
\hline \multicolumn{6}{|l|}{ Age group (y) } \\
\hline & 1 & $50-59$ vs $<50$ & .395 & 1.487 & $0.596-3.712$ \\
\hline & 2 & $60-69$ vs $<50$ & .221 & 1.718 & $0.722-4.086$ \\
\hline & 2 & $70-79$ vs $<50$ & .128 & 1.940 & $0.826-4.558$ \\
\hline & 3 & $\geq 80$ vs $<50$ & $<.001$ & 2.689 & $1.123-6.441$ \\
\hline
\end{tabular}

$O R$, Odds ratio; $C I$, confidence interval. *History of each comorbid disease as defined in the CCI. †Nonelective surgery includes urgent and emergency surgery as defined by the Society of Thoracic Surgeons. ‡๋djusted for all other factors in the model.

were independent predictors of nonelective repair in multivariate analysis. Patients who had a nonelective operation were also significantly more likely to have a planned open repair $(23 / 199 ; 12 \%)$ than patients who underwent elective repair $(6 / 781 ; 0.8 \%[P<.001])$.

\section{DISCUSSION}

By using known pretreatment covariates, we have developed clinical prediction rules for mortality and major morbidity after GPEH repair that provide good discriminatory accuracy and could be easily implemented in the clinical setting. We found that the combination of nonelective operation, age 80 years or more, history of pulmonary disease,

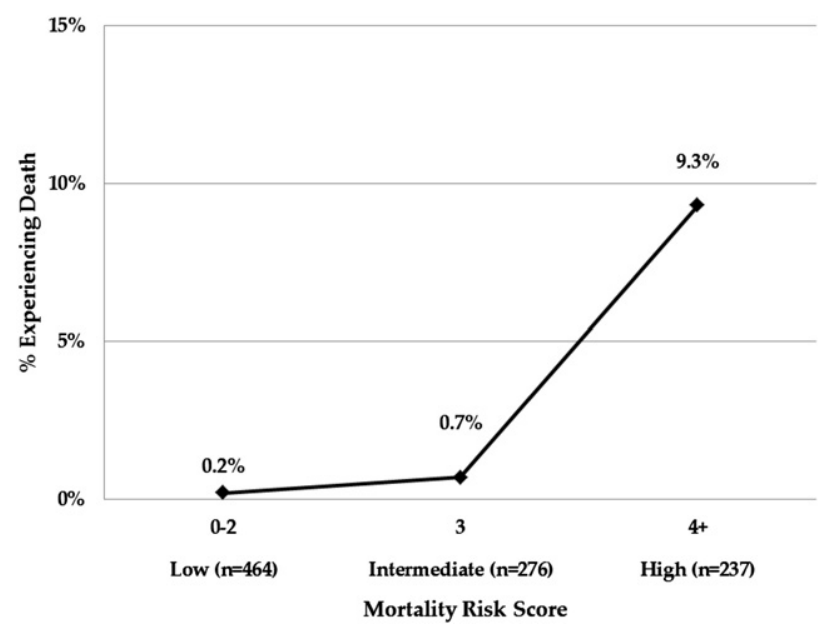

FIGURE 1. In-hospital or 30-day mortality. Patients were assigned points according to the presence of risk factors for mortality. Increasing risk score correlates with the incidence of mortality. Incidence of mortality (in bold) and numbers of patients within each group are shown. and history of congestive heart failure clearly identified a group of patients at increased risk for perioperative mortality. By using these variables, our models also identified a group of patients at very low risk for perioperative mortality. Likewise, our 5-covariate model for major morbidity, including sex, age at operation, urgency of operation, history of congestive heart failure, and history of pulmonary disease, facilitated assignment of patients into minimal-, low-, intermediate-, and high-risk categories on the basis of their pretreatment scores. Of note, the risk for perioperative mortality more than tripled and the risk for major morbidity more than doubled when nonelective repair was required. Although a limited number of previous reports have examined the association of preoperative variables to adverse outcomes after GPEH repair, ${ }^{2,22}$ our study is the first to develop prediction models for adverse outcome based on pretreatment variables.

\section{Impact of Nonelective Repair on Patient Risk}

There is little debate that the risks of operative intervention outweigh the benefits in completely asymptomatic patients with GPEH. In symptomatic patients, however, the literature is somewhat conflicting. Historically, it has been recommended that all patients with paraesophageal hernia undergo repair at the time of diagnosis to prevent the catastrophic complications that can occur with acute mechanical symptoms, including gastric strangulation, massive hemorrhage, or perforation. ${ }^{13}$ More recently, however, it was suggested that minimally symptomatic patients can be managed expectantly. ${ }^{10}$ By using Markov modeling, Stylopoulos and colleagues ${ }^{10}$ created theoretic cohorts with minimal symptoms and determined the outcomes for 2 approaches: immediate repair or watchful waiting. In their decision analysis, the mortality difference between elective 
TABLE 3. Distribution of postoperative morbidity within categories of predicted risk

\begin{tabular}{|c|c|c|c|c|c|c|}
\hline \multirow{4}{*}{ Postoperative outcome: $\mathbf{n}(\%)$} & \multirow{4}{*}{$\begin{array}{c}\text { Total } \\
\mathbf{n}=\mathbf{9 8 0}\end{array}$} & \multicolumn{4}{|c|}{ Major morbidity risk score } & \multirow[b]{4}{*}{$P$ value } \\
\hline & & \multirow{3}{*}{$\frac{\frac{\text { Minimal }}{0-2}}{n=407}$} & \multirow{3}{*}{$\frac{\text { Low }}{\frac{3}{n=173}}$} & \multirow{3}{*}{$\frac{\frac{\text { Intermediate }}{4}}{n=188}$} & \multirow{3}{*}{$\frac{\text { High }}{\frac{5+}{n=212}}$} & \\
\hline & & & & & & \\
\hline & & & & & & \\
\hline At least 1 major adverse outcome & $224(23)$ & $55(13.5)$ & $34(19.7)$ & $49(26.1)$ & $86(40.6)$ & $<.001$ \\
\hline Any pulmonary complication* & $199(20.3)$ & $51(12.5)$ & $28(16)$ & $38(20)$ & $82(39)$ & $<.001$ \\
\hline Pneumonia & $65(6.7)$ & $15(3.7)$ & $7(4.1)$ & $14(7.5)$ & $29(13.8)$ & $<.001$ \\
\hline Reintubation & $42(4.3)$ & $7(1.7)$ & $3(1.7)$ & $11(5.9)$ & $21(9.9)$ & $<.001$ \\
\hline Postoperative tracheostomy & $8(0.8)$ & $1(0.25)$ & $0(0)$ & $2(1.1)$ & $5(2.4)$ & .027 \\
\hline Pulmonary embolism & $34(3.5)$ & $13(3.2)$ & $6(3.5)$ & $8(4.3)$ & $7(3.3)$ & .909 \\
\hline Myocardial infarction & $11(1.1)$ & $3(0.74)$ & $0(0)$ & $1(0.5)$ & $7(3.3)$ & .012 \\
\hline Congestive heart failure & $36(3.7)$ & $2(0.5)$ & $4(2.3)$ & $5(2.7)$ & $25(11.9)$ & $<.001$ \\
\hline Acute renal failure & $25(2.6)$ & $0(0)$ & $3(1.7)$ & $3(1.6)$ & $19(9)$ & $<.001$ \\
\hline Cerebral vascular accident & $6(0.6)$ & $1(0.25)$ & $0(0)$ & $1(0.53)$ & $4(1.9)$ & .068 \\
\hline Septic shock or bacteremia & $21(2.1)$ & $2(0.5)$ & $3(1.7)$ & $7(3.7)$ & $9(4.3)$ & .003 \\
\hline Postoperative gastric or esophageal leak & $21(2.2)$ & $6(1.5)$ & $2(1.2)$ & $7(3.7)$ & $6(2.8)$ & .229 \\
\hline Perioperative hernia recurrence & $12(1.2)$ & $4(1)$ & $3(1.7)$ & $1(0.5)$ & $4(1.9)$ & .528 \\
\hline Readmission within $30 \mathrm{~d}$ of operation & $96(9.8)$ & $21(5.2)$ & $16(9.3)$ & $22(11.7)$ & $37(17.5)$ & $<.001$ \\
\hline Reoperation within $30 \mathrm{~d}$ of operation & $60(6.1)$ & $13(3.2)$ & $8(4.7)$ & $17(9.0)$ & $22(10.4)$ & .001 \\
\hline
\end{tabular}

*Any pulmonary complication includes pneumonia, tracheostomy, unplanned bronchoscopy, pneumothorax or pleural effusion requiring drainage, reintubation, and prolonged initial ventilation more than 48 hours.

and emergency surgery was the only factor influencing the value of elective surgery. They concluded that, assuming a $17 \%$ mortality rate of emergency surgery, elective laparoscopic GPEH repair becomes the optimal management if mortality with elective laparoscopic repair is less than $1 \%$. If the mortality rate of emergency surgery is $6 \%$, a value close to the mortality rate of $8 \%$ seen in our series, elective repair is recommended only if it can be achieved with mortality of $0.5 \%$ or less. ${ }^{10}$

When considering whether to adopt the recommendations for watchful waiting into clinical practice, it is important to note that the model developed by Stylopoulos and colleagues ${ }^{10}$ was designed to establish mortality cutoffs for minimally symptomatic patients. In this model, minimally symptomatic was defined as patients with symptoms (belching and heartburn) that do not affect the quality of life of the patient. ${ }^{10}$ However, we and others have reported the significant impact of GPEH on quality of life, even in patients whose primary symptom is heartburn. ${ }^{1,3,5,9,23-28}$ The majority of patients, particularly those with larger hernias, have mechanical symptoms such as pain, postprandial bloating, and bleeding (acute and occult). Careful assessment for symptoms frequently reveals symptoms of chest and abdominal pain, postprandial bloating, dysphagia, chronic anemia, weight loss, change in eating habits, or food avoidance. ${ }^{6,7,9}$ In patients with any of these symptoms, particularly when $75 \%$ or more of the stomach is herniated into the mediastinum, careful consideration for elective repair should be entertained.

In the current study, we found a strong independent association of nonelective repair with increased risk of mortality and major morbidity after GPEH repair. This is consistent with many other reports in the literature; recently published analyses of administrative datasets show mortality for emergency repair ranges from $5.1 \%$ to $16.4 \% \cdot{ }^{10,12,15}$ It has also been noted that the risk of nonelective repair increases dramatically in the very elderly, as does the risk for associated mortality. Poulose and colleagues ${ }^{14}$ assessed the impact of nonelective repair on postoperative mortality in octogenarians using the 2005 National Inpatient Sample; they found that, similar to the results presented in our series, $43 \%$ of the octogenarians in the nationwide sample underwent nonelective repair ( $47 \%$ in our series). Mortality after nonelective repair in the nationwide sample was markedly higher than for nonelective repair (16\% for nonelective vs $2.4 \%$ for elective repair). We also found a marked increase in mortality $(14 \%)$ for nonelective repair in the octogenarians compared with $6 \%$ for elective repair, although it did not reach a statistical significance level because of the smaller numbers of patients. Nonelective repair has also been shown to be associated with longer hospital stay and higher cost. ${ }^{12}$

\section{Impact of Increasing Age, Comorbidity, and Hernia Size at Time of Repair on Patient Risk}

Despite the higher risk, age alone should not be a contraindication to elective laparoscopic repair in symptomatic patients, because the majority of elderly patients do not experience postoperative mortality or major morbidity. ${ }^{2,11,15,22,27,29,30}$ In addition to age, a history of congestive heart failure requiring medical therapy and a history of pulmonary disease are predictors of adverse outcome after GPEH repair. Although none of the electively repaired patients in this current study had 
TABLE 4. Association of patient demographics, comorbid diseases, and symptoms with urgent or emergency paraesophageal hernia repair

\begin{tabular}{|c|c|c|c|c|c|c|c|}
\hline \multirow{3}{*}{ Patient demographics: $n(\%)$} & & \multirow{3}{*}{$\begin{array}{l}\text { Overall } \\
\mathbf{n}=980\end{array}$} & \multicolumn{2}{|c|}{ Nonelective operation } & \multirow[b]{3}{*}{$P$ value } & \multirow[b]{3}{*}{ Adjusted $\mathbf{O R} \S$} & \multirow[b]{3}{*}{$\mathbf{9 5} \% \mathrm{CI}$} \\
\hline & & & \multirow{2}{*}{$\begin{array}{l}\text { Elective } \\
\mathrm{n}=781\end{array}$} & \multirow{2}{*}{$\begin{array}{c}\text { Nonelective } \\
\mathrm{n}=199 \\
\end{array}$} & & & \\
\hline & & & & & & & \\
\hline \multirow[t]{2}{*}{ Sex } & Female & $733(75)$ & $595(81)$ & $138(19)$ & .055 & & \\
\hline & Male & $247(25)$ & $186(75)$ & $61(25)$ & & & \\
\hline \multirow[t]{5}{*}{ Age group at operation } & $<50 \mathrm{y}$ & $60(6)$ & $51(85)$ & $9(15)$ & $<.001$ & ref & ref \\
\hline & $50-59$ y & $148(15)$ & $130(88)$ & $18(12)$ & & 0.80 & $(0.33-1.96)$ \\
\hline & $60-69$ y & $261(27)$ & $234(90)$ & $27(10)$ & & 0.61 & $(0.26-1.42)$ \\
\hline & $70-79 \mathrm{y}$ & $319(33)$ & $265(83)$ & $55(17)$ & & 0.93 & $(0.42-2.09)$ \\
\hline & $\geq 80 \mathrm{y}$ & $192(20)$ & $102(53)$ & $90(47)$ & & 2.66 & $(1.16-6.10)$ \\
\hline \multirow[t]{5}{*}{ BMI by WHO classification* } & Underweight & $16(2)$ & $9(56)$ & $7(44)$ & $<.001$ & ref & ref \\
\hline & Ideal & $185(19)$ & $126(68)$ & $59(32)$ & & 0.50 & $(0.13-1.73)$ \\
\hline & Overweight & 356 (37) & $295(83)$ & $61(17)$ & & 0.35 & $(0.10-1.12)$ \\
\hline & Obese & $258(27)$ & $220(85)$ & $38(15)$ & & 0.36 & $(0.10-1.27)$ \\
\hline & Severely obese & $149(15)$ & $127(85)$ & $22(15)$ & & 0.42 & $(0.12-1.54)$ \\
\hline \multirow[t]{4}{*}{ Preoperative hernia size $(\%) \dagger$} & $30 \%-49 \%$ & $174(18)$ & $157(90)$ & $17(10)$ & $<.001$ & ref & ref \\
\hline & $50 \%-74 \%$ & $354(36)$ & $300(85)$ & $54(15)$ & & 1.40 & $(0.74-2.60)$ \\
\hline & $75 \%-99 \%$ & $212(22)$ & $159(75)$ & $53(25)$ & & 2.60 & $(1.25-4.88)$ \\
\hline & Intrathoracic stomach & $240(24)$ & $165(69)$ & $75(31)$ & & 3.00 & $(1.59-5.58)$ \\
\hline \multicolumn{8}{|c|}{ History of pretreatment Charlson-defined patient comorbidity } \\
\hline \multirow[t]{2}{*}{ Myocardial infarction or revascularization } & No & $833(85)$ & $677(81)$ & $156(19)$ & .005 & 1.27 & $(0.80-2.02)$ \\
\hline & Yes & $147(15)$ & $104(71)$ & $43(29)$ & & & \\
\hline \multirow[t]{2}{*}{ Congestive heart failure } & No & $941(96)$ & $761(81)$ & $180(19)$ & $<.001$ & 1.84 & $(0.83-4.05)$ \\
\hline & Yes & $39(4)$ & $20(51)$ & $19(48)$ & & & \\
\hline \multirow[t]{2}{*}{ Cerebral vascular accident or transient attack } & No & $904(92)$ & $737(82)$ & $167(18)$ & $<.001$ & 1.60 & $(0.87-2.83)$ \\
\hline & Yes & $76(8)$ & $44(58)$ & $32(42)$ & & & \\
\hline \multirow[t]{2}{*}{ Dementia } & No & $937(96)$ & $763(81)$ & $174(19)$ & $<.001$ & 2.43 & $(1.13-5.22)$ \\
\hline & Yes & $43(4)$ & $18(42)$ & $25(58)$ & & & \\
\hline \multirow[t]{2}{*}{ Pulmonary disease } & No & $690(70)$ & $550(80)$ & $140(20)$ & 1.000 & & \\
\hline & Yes & $290(30)$ & $231(80)$ & $59(20)$ & & & \\
\hline \multirow[t]{2}{*}{ Connective tissue disorder } & No & $942(96)$ & $750(80)$ & $192(20)$ & 1.000 & & \\
\hline & Yes & $38(4)$ & $31(82)$ & 7 (18) & & & \\
\hline Peptic ulcer disease & No & $794(81)$ & $655(82)$ & $139(18)$ & $<.001$ & 2.20 & $(1.50-3.30)$ \\
\hline & Yes & $186(19)$ & $126(68)$ & $60(32)$ & & & \\
\hline Diabetes requiring medical therapy & No & $909(93)$ & $728(80)$ & $181(20)$ & .284 & & \\
\hline & Yes & $71(7)$ & $53(75)$ & $18(25)$ & & & \\
\hline Malignancy in the past $5 \mathrm{y}$ & No & $924(94)$ & $740(80)$ & $184(20)$ & .231 & & \\
\hline & Yes & $56(6)$ & $41(73)$ & $15(27)$ & & & \\
\hline Rare CCI variables $\ddagger$ & No & $939(96)$ & $753(80)$ & $186(20)$ & .074 & & \\
\hline & Yes & $41(4)$ & $28(68)$ & $13(32)$ & & & \\
\hline Preoperative symptoms & & & & & & & \\
\hline Respiratory symptoms & No & $232(34)$ & $164(71)$ & $68(29)$ & $<.001$ & & \\
\hline & Yes & $450(66)$ & $373(83)$ & $77(17)$ & & & \\
\hline Chest or abdominal pain & No & $273(32)$ & $236(86)$ & 37 (14) & .001 & & \\
\hline & Yes & $589(68)$ & $449(76)$ & $140(24)$ & & & \\
\hline Heartburn & No & $335(35)$ & $226(67)$ & $109(33)$ & $<.001$ & & \\
\hline & Yes & $626(65)$ & $545(87)$ & $81(13)$ & & & \\
\hline Dysphagia & No & $533(55)$ & $418(78)$ & $115(22)$ & .122 & & \\
\hline & Yes & $431(45)$ & $356(83)$ & 75 (17) & & & \\
\hline Regurgitation & No & 377 (39) & $316(84)$ & $61(16)$ & .014 & & \\
\hline & Yes & $590(61)$ & $456(77)$ & $134(23)$ & & & \\
\hline Bloating & No & $619(66)$ & $483(78)$ & $136(22)$ & .016 & & \\
\hline & Yes & $325(34)$ & $275(85)$ & $50(15)$ & & & \\
\hline
\end{tabular}

$O R$, Odds ratio; $C I$, confidence interval; $B M I$, body mass index $\left(\mathrm{kg} / \mathrm{m}^{2}\right) ; W H O$, World Health Organization; $C C I$, Charlson Comorbidity Index. *Underweight BMI $<18.5$, ideal BMI 18.5-25, overweight BMI 25-30, obese BMI 30-35, severely obese BMI $\geq 35$. $\nmid$ Percent of stomach within the mediastinum by barium esophagram, preoperative endoscopy, or intraoperative description. $\ddagger$ Rare CCI variables ( $<3 \%$ of cohort) include peripheral vascular disease, renal disease, liver disease, diabetes mellitus with organ damage, hemiplegia or paraplegia, lymphoma, leukemia, metastatic cancer, and acquired immunodeficiency syndrome. §Each adjusted for the other variables in the model (age group, BMI, size of hernia, myocardial infarction, congestive heart failure, cerebral vascular accident or transient attack, dementia, and peptic ulcer disease). 
decompensated congestive heart failure, awareness of the increased risk for adverse outcome associated with these comorbid conditions would allow for medical optimization before elective surgery for symptomatic GPEH. Recognition of the increased risk may also inform the decision to forego surgical repair altogether if the hernia is small, the patient is very elderly with other comorbid conditions, and the symptoms are predominantly regurgitation related rather than obstructive.

To address these risk factors, our current approach to patients with GPEH is routine cardiac testing on the majority of patients before elective and urgent repair if the patient's clinical status will allow. This is because the majority of patients are elderly and have a history of smoking or obesity or other cardiac risk factors, such as family history, personal history of cardiac disease, hypertension, hyperlipidemia, or diabetes mellitus. In addition, chest pain is often one of the primary symptoms. Although the chest pain is often due to the GPEH itself, there are occasional patients in whom concomitant coronary artery disease is identified. For those patients in whom repair is elective and coronary disease is identified, optimization with medical or invasive techniques is performed before GPEH repair. It is important to note that all patients who are under the management of a cardiologist are sent to their provider for preoperative optimization and clearance. Pulmonary function testing is obtained on any patients with pulmonary symptoms, and smoking cessation is strongly encouraged for current smokers. This proactive approach likely reduces postoperative mortality and major morbidity and improves outcomes for these patients.

The clinical prediction rules presented may provide a more accurate understanding of patient-specific risk for adverse outcomes with elective repair at an earlier age compared with the risk of nonelective repair when older if watchful waiting is undertaken. For example, a 65-yearold woman with pulmonary disease and GPEH would have a risk score for mortality of 3 (mortality rate, $0.7 \%$ ) if the operation was performed electively and a risk score of 6 (mortality rate, 9.3\%) if performed nonelectively. With regard to major postoperative morbidity, the patient's current risk category for elective repair is intermediate (risk score 4 for age and pulmonary disease). Waiting for acute presentation and nonelective repair increases the risk category for major morbidity to high risk $(40 \%)$. Given an estimated lifetime risk of developing acute symptoms of $18 \%$ and an estimated annual probability of symptom progression of approximately $14 \%,{ }^{10}$ elective repair would be warranted, preferably before the patient reaches 80 years of age, when perioperative mortality increases steeply.

Although paraesophageal hernia size did not remain in the final model because of high collinearity with age and nonelective operation, it is important to note that the risk of nonelective operation did increase with increased hernia size. Combined with the other high-risk criteria in the clinical prediction models, waiting for an acute presentation in a patient with the majority of stomach herniated into the mediastinum could prove fatal. On the other hand, a patient with other high-risk criteria and a small GPEH may be advised to pursue medical therapy, especially if the symptoms are predominantly heartburn.

\section{Study Strengths and Limitations}

Our study has several strengths and limitations. With the large numbers of patients in our extensive, prospectively maintained database for GPEH, and definition-driven abstraction of covariates, we are uniquely positioned to develop these clinical predication rules. However, our study findings may be limited by the fact that the overall mortality in our series was low and restricted our ability to identify additional predictors of mortality, such as stratifying risk by expanded age groups. Thus, our model may have overlooked other important predictors. Our findings may be hard to generalize because our center has extensive expertise in the management of GPEH and the majority of both elective and nonelective repairs were performed laparoscopically. It is possible that open repair is a significant predictor of adverse outcome after GPEH repair; this could not be assessed in our series because of the significant collinearity between nonelective repair and a planned open approach to operation. As a result, the rate of adverse outcomes in our series may not represent the observed rates in other centers.

Ideally, our model would have also undergone external validation; however, we were able to perform internal cross-validation bootstrapping using the leave-one-out strategy, which showed error rates that were similar to the rates for the developed models for both outcomes. Further validation of the models in other settings is clearly required. Finally, the patients' goals for symptom relief and improved quality of life are also important considerations in guiding the decision to offer surgery.

\section{CONCLUSIONS}

We have developed clinical prediction rules for postoperative mortality and major morbidity after GPEH repair in a high-volume center with extensive expertise in the management of GPEH that have good discriminatory accuracy and facilitate assignment of patients to risk categories for adverse outcome. Our models included patient age at operation, sex, urgency of surgery, congestive heart failure, and pulmonary disease. After further refinement and prospective validation, these clinical prediction rules for mortality and major morbidity after GPEH repair could provide patient-specific risk prediction, tailored for individual patient characteristics, and contribute to decision-making regarding surgical intervention.

The authors thank Sunee Hempel, Megan Lunz, Kathy Lovas, and Shannon Wyszomierski for expert data abstraction, database 
management, and editorial assistance. Without their dedication, this work would not be possible.

\section{References}

1. Luketich JD, Nason KS, Christie NA, et al. Outcomes after a decade of laparoscopic giant paraesophageal hernia repair. J Thorac Cardiovasc Surg. 2010;139: 395-404.e1.

2. Larusson HJ, Zingg U, Hahnloser D, Delport K, Seifert B, Oertli D. Predictive factors for morbidity and mortality in patients undergoing laparoscopic paraesophageal hernia repair: age, ASA score and operation type influence morbidity. World J Surg. 2009;33:980-5.

3. Nason KS, Luketich JD, Awais O, et al. Quality of life after collis gastroplasty for short esophagus in patients with paraesophageal hernia. Ann Thorac Surg. 2011; 92:1854-61.

4. Targarona EM, Novell J, Vela S, et al. Mid term analysis of safety and quality of life after the laparoscopic repair of paraesophageal hiatal hernia. Surg Endosc. 2004; 18:1045-50.

5. Parameswaran R, Ali A, Velmurugan S, Adjepong SE, Sigurdsson A. Laparoscopic repair of large paraesophageal hiatus hernia: quality of life and durability. Surg Endosc. 2006;20:1221-4.

6. Hayden JD, Jamieson GG. Effect on iron deficiency anemia of laparoscopic repair of large paraesophageal hernias. Dis Esophagus. 2005;18:329-31.

7. Low DE, Simchuk EJ. Effect of paraesophageal hernia repair on pulmonary function. Ann Thorac Surg. 2002;74:333-7.

8. Moskovitz M, Fadden R, Min T, Jansma D, Gavaler J. Large hiatal hernias, anemia, and linear gastric erosion: studies of etiology and medical therapy. Am J Gastroenterol. 1992;87:622-6.

9. Carrott PW, Hong J, Kuppusamy M, Kirtland S, Koehler RP, Low DE. Repair of giant paraesophageal hernias routinely produces improvement in respiratory function. J Thorac Cardiovasc Surg. 2012;143:398-404.

10. Stylopoulos N, Gazelle GS, Rattner DW. Paraesophageal hernias: operation or observation? Ann Surg. 2002;236:492-501.

11. Polomsky M, Jones CE, Sepesi B, et al. Should elective repair of intrathoracic stomach be encouraged? J Gastrointest Surg. 2010;14:203-10.

12. Polomsky M, Hu R, Sepesi B, et al. A population-based analysis of emergent vs elective hospital admissions for an intrathoracic stomach. Surg Endosc. 2010;24: 1250-5.

13. Hill LD. Incarcerated paraesophageal hernia. A surgical emergency. Am J Surg. 1973;126:286-91.

14. Poulose BK, Gosen C, Marks JM, et al. Inpatient mortality analysis of paraesophageal hernia repair in octogenarians. J Gastrointest Surg. 2008;12:1888-92.
15. Sihvo EI, Salo JA, Rasanen JV, Rantanen TK. Fatal complications of adult paraesophageal hernia: a population-based study. J Thorac Cardiovasc Surg. 2009; 137:419-24

16. Reilly BM, Evans AT. Translating clinical research into clinical practice: impact of using prediction rules to make decisions. Ann Intern Med. 2006;144:201-9.

17. Fine MJ, Auble TE, Yealy DM, et al. A prediction rule to identify low-risk patients with community-acquired pneumonia. N Engl J Med. 1997;336:243-50.

18. Pearson FG, Cooper JD, Ilves R, Todd TR, Jamieson WR. Massive hiatal hernia with incarceration: a report of 53 cases. Ann Thorac Surg. 1983;35:45-51.

19. Nason KS, Luketich JD, Witteman BP, Levy RM. The laparoscopic approach to paraesophageal hernia repair. J Gastrointest Surg. 2012;16:417-26.

20. Charlson ME, Pompei P, Ales KL, MacKenzie CR. A new method of classifying prognostic comorbidity in longitudinal studies: development and validation. $J$ Chronic Dis. 1987;40:373-83.

21. Society of Thoracic Surgeons. STS General Thoracic Data Specifications, Version 2.081. Society of Thoracic Surgeons General Thoracic Surgery National Database; 2011.

22. Gupta A, Chang D, Steele KE, Schweitzer MA, Lyn-Sue J, Lidor AO. Looking beyond age and co-morbidities as predictors of outcomes in paraesophageal hernia repair. J Gastrointest Surg. 2008;12:2119-24.

23. Mark LA, Okrainec A, Ferri LE, Feldman LS, Mayrand S, Fried GM. Comparison of patient-centered outcomes after laparoscopic Nissen fundoplication for gastroesophageal reflux disease or paraesophageal hernia. Surg Endosc. 2008; 22:343-7.

24. Hazebroek EJ, Gananadha S, Koak Y, Berry H, Leibman S, Smith GS. Laparoscopic paraesophageal hernia repair: quality of life outcomes in the elderly. Dis Esophagus. 2008;21:737-41.

25. Velanovich V, Karmy-Jones R. Surgical management of paraesophageal hernias: outcome and quality of life analysis. Dig Surg. 2001;18:432-8.

26. Louie BE, Blitz M, Farivar AS, Orlina J, Aye RW. Repair of symptomatic gian paraesophageal hernias in elderly ( $>70$ years) patients results in improved quality of life. J Gastrointest Surg. 2011;15:389-96.

27. Gangopadhyay N, Perrone JM, Soper NJ, et al. Outcomes of laparoscopic paraesophageal hernia repair in elderly and high-risk patients. Surgery. 2006;140: 491-9.

28. Nason KS, Luketich JD, Qureshi I, et al. Laparoscopic repair of giant paraesophageal hernia results in long-term patient satisfaction and a durable repair. $J$ Gastrointest Surg. 2008;12:2066-77.

29. Hosking MP, Warner MA, Lobdell CM, Offord KP, Melton LJ III. Outcomes of surgery in patients 90 years of age and older. JAMA. 1989;261:1909-15.

30. Kojima Y, Narita M. Postoperative outcome among elderly patients after general anesthesia. Acta Anaesthesiol Scand. 2006;50:19-25. 